\title{
Analysis of Prognostic Factors and Design of Prognosis Model for Patients with Stage IV Gastric Cancer Following First-Line Palliative Chemotherapy
}

This article was published in the following Dove Press journal:

Cancer Management and Research

\author{
Qiyin Zhou* \\ Xi Lan (iD $*$ \\ $\mathrm{Ni} \mathrm{Li}$ \\ Daozu Yuan \\ Jiliang Zhang \\ Department of Oncology, Chengdu \\ Seventh People Hospital (Chengdu \\ Tumorous Disease Quality Control \\ Center), Chengdu 6I0000, People's \\ Republic of China \\ *These authors contributed equally to \\ this work
}

Background: This study was to investigate the prognostic factors of patients with advanced gastric cancer and described a sample model to better differentiate the patients who could better benefit from palliative chemotherapy.

Patients and Methods: In this retrospective study, 112 gastric cancer patients at stage IV following first-line chemotherapy were enrolled from July 2013 to September 2019. The clinical factors including age, sex, ECOG, pathologic types, metastatic sites, blood indexes, response of first-line chemotherapy, and survival were collected. The treatment responses were evaluated using the response evaluation criteria in solid tumors (RECIST). The survival curves were drawn by the Kaplan-Meier method, and the independent prognostic factors of overall survival (OS) were analyzed by Cox proportional hazards regression model.

Results: In this study, the median overall survival (mOS) of gastric cancer patients was 10.5 months, the disease remission rate (PR) was $21.4 \%$, and the disease control rate (DCR) was $86.6 \%$. Multivariate analysis identified 5 independent prognostic factors: peritoneal metastasis $[P=0.002$; hazard risk (HR), 2.394; 95\% CI 1.394-4.113], hemoglobin $<90 \mathrm{~g} / \mathrm{L}[P=$ 0.001; hazard risk (HR), 2.674; 95\% CI 1.536-4.655], LDH $\geq 225 \mathrm{U} / \mathrm{L}[P=0.033$; hazard risk (HR), 1.818; 95\% CI 1.409-3.150], and 3 times higher level of CEA $[P=0.006$; hazard risk (HR), 2.123; 95\% CI 1.238-3.640] along with CA199 [P=0.005; hazard risk (HR), $2.544 ; 95 \%$ CI 1.332-4.856] than upper limit of normal. Based on the obtained data, a prognostic index was constructed, dividing the patients into three risk groups: low $(n=$ $67)$, intermediate $(n=35)$, and high-risk group $(n=10)$. The mOS for low, intermediate, and high-risk groups was 13.9 months (95\% CI 10.7-17.1), 8.1 months (95\% CI 5.7-10.4), and 3.9 months (95\% CI 2.6-5.3), respectively, whereas the 1 -year survival rate was $56.4 \%$, $20.0 \%$, and $0.0 \%$, respectively $(P<0.001)$.

Conclusion: This model should facilitate the prediction of treatment outcomes and then individualized treatment of advanced gastric cancer patients.

Keywords: prognostic factor, advanced gastric cancer, prognosis model, first-line chemotherapy

\section{Introduction}

Gastric cancer is one of the most common malignant tumors, which is in the top three of mortality rate among malignant tumors. ${ }^{1}$ Due to its insidious onset and the absence of special symptoms in its initial stage, most patients are diagnosed at later
Correspondence: Jiliang Zhang Department of Oncology, Chengdu Seventh People Hospital (Chengdu Tumorous Disease Quality Control Center), 5I Zhimin Road, Chengdu 610000, People's Republic of China Tel +8613982070262

Email Zhangjiliang126@I26.com 
stages and cannot be radically operated, ${ }^{2}$ especially in China. The current treatments include palliative gastrectomy, radiotherapy, chemotherapy, molecular targeted therapy, and immunotherapy. At present, chemotherapy is still the main treatment for gastric cancer patients with localadvanced or tumor recurrence or metastasis, but 5-year overall survival (OS) rate rarely exceeds $5 \%$ of all treated patients. Although trastuzumab in combination with chemotherapy can prolong the survival of patients with HER2-positive advanced gastric cancer, ${ }^{3-5}$ several studies show that the HER2-positive rate of gastric cancer patients in China is only around $12 \%-13 \%{ }^{6,7}$ The role of chemotherapy is still of paramount importance in treatment of HER2-negative gastric cancer. In a sense, chemotherapy can prolong the survival time and improve the life quality of these patients, ${ }^{8}$ but the response rate of first-line chemotherapy for advanced gastric cancer patients is no more than $50 \%$, and the median overall survival (mOS) is about 13 to 16 months, ${ }^{9,10}$ which is not expected in the clinical setting. It appears that not all gastric cancer patients at stage IV could benefit from palliative chemotherapy, thus it is very important to choose the right patients for chemotherapy.

Prognostic parameters play an important role in the implementation of risk-based stratification of therapy in patients with advance gastric cancer. Till now, a multitude of indicators on survival prognosis for advanced gastric cancer patients receiving first-line chemotherapy have been reported by scholars both in China and abroad, including performance status (PS), hepatic metastases, ascites, number of metastases, hemoglobin, serum albumin, alkaline phosphatase, serum CEA, serum CA199, etc. ${ }^{11-14}$ Besides, as a key factor of targeted therapy, the expression of HER2 has not been found to be correlated with prognosis of gastric cancer patients in a number of large-sample studies in China and abroad. ${ }^{6,15-17}$ Some other studies show that HER2 may affect the survival of gastric cancer patients in early stage, ${ }^{18,19}$ but has no effect on the prognosis of patients in late stage. ${ }^{18,20,21}$ However, few studies have combined the indicators such as performance status, blood routine, biochemistry, and tumor markers to comprehensively evaluate the prognosis of patients. In this study, we firstly integrated the results of previous studies and included some new influence factors to analyze the prognosis of patients with metastatic gastric carcinoma. Taking the studies about prognostic analysis of advanced gastric cancer patients undergoing second-line chemotherapy reported by Catalano et al and Kanagavel et al as reference, ${ }^{22,23}$ we then designed a simple prognostic model based on the independent predictors obtained by multivariate analysis to stratify these patients into different risk groups. This will allow us to better differentiate patient populations and to better predict treatment outcomes.

\section{Cases Collectors}

The inclusion criteria for cases were: (i) pathologically proven gastric carcinoma with measurable or evaluable lesions; (ii) evidence of unresectable disease like malignant ascites or organ metastasis confirmed by cytological diagnosis or imaging tests; (iii) no prior surgical therapy, chemotherapy, or radiotherapy after recurrence; (iv) no less than 2 cycles of first-line palliative chemotherapy without target drugs (such as trastuzumab, etc.) in combination; (v) performance status (PS) was in the range of $0-2$ at the time of the first-line chemotherapy. We firstly assessed the general condition and clinicopathological characteristics of these patients and then collected the fasting indicators in blood one week before chemotherapy including hemoglobin, platelet, serum LDH, serum CEA, and serum CA199. The chemotherapy regimens were fluorouracil alone or in combination with other agents which consisted of the following: (i) docetaxel or paclitaxel, 5-fluorouracil (5-FU) $(n=25)$; (ii) oxaliplatin or irinotecan, leucovorin, 5-FU (modified FOLFOX or FOLFIRI) ( $n=40)$; (iii) cisplatin, 5-FU (PF) $(n=13)$; (iv) oxaliplatin, capecitabine (XELOX) $(n=10)$; (v) oxaliplatin, S-1 (SOX) ( $n=11)$; (vi) epirubicin, cisplatin, 5-FU (ECF) $(n=5)$; (vii) 5-FU or capecitabine or S-1 $(n=8)$. RECIST standard was used to evaluate the efficacy of chemotherapy after two cycles. The period from the date of diagnosis of advanced gastric cancer to the date of death or the last follow-up visit was defined as the OS, which was the primary end point of the study. All patients were followedup (outpatient or telephone) every 3 months in that period.

\section{Methods}

Statistical analysis: SPSS19.0 software was used for data processing and analysis, and $P<0.05$ was considered statistically significant. Univariate survival analysis was performed by the Kaplan-Meier method and was tested by log-rank method. Multivariate analyses were carried out using the Cox proportional hazards model. 


\section{Results}

\section{Patient Characteristics}

From July 2013 to September 2019, a total of 112 patients with metastatic, unresectable, or recurrent gastric cancer in Chengdu Seventh People Hospital (Chengdu Tumorous Disease Quality Control Center) enrolled in our study, including 34 females and 78 males, and the median age was 57 with a range of 28-78. Until the closing date of December 31, 2019, a total of 73 patients died and 3 were lost to follow-up. As shown in Table 1, approximately $66 \%$ (74 of 112) of patients had Eastern Cooperative Oncology Group (ECOG) 0-1 at the time of the first-line palliative chemotherapy. In terms of pathological types, adenocarcinoma accounted for about 83\% (93 of 112), and, in terms of tumor grade, poor differentiation accounted for nearly $62 \%$ (69 of 112). Distant metastases were found in all cases, and liver metastases or ascites were present in about $30 \%$ and nearly $40 \%$ of patients, respectively. The hemoglobin level of 23 (20.5\%) patients was lower than $90 \mathrm{~g} / \mathrm{L}$, and the lactate dehydrogenase (LDH) level was above $225 \mathrm{U} / \mathrm{L}$ in 36 (32.1\%) patients. A total of $69(61.8 \%)$ patients had a level of CEA three times higher than upper limit of normal $(4.7 \mathrm{ng} / \mathrm{mL})$. Similarly, 21 (18.7\%) patients had a level of CA199 three times higher than upper limit of normal $(27 \mathrm{U} / \mathrm{mL})$. About the treatment response, there were no complete response (CR) cases and $24(21.4 \%)$ partial response (PR) cases, giving a disease control rate (DCR) of $86.6 \%$.

\section{Univariate and Multivariate Analysis}

In the study, the median OS of all patients was 10.5 months [95\% confidence interval (CI) 8.8- 12.2], and the 1-year survival rate was $38.4 \%$. There were thirteen factors included in the univariate analysis (Table 2): age, gender, performance status, pathologic types, histologic grade, number of metastatic organs, hemoglobin level, platelet count, LDH level, CEA level, CA199 level, and metastasis to liver and to peritoneum. Among them, six factors showed statistically significant association with the mOS of metastatic gastric cancer patients and were further delineated by multivariate Cox proportional hazards model, including ECOG PS of $2[P=0.449$; hazard risk (HR), 1.246 ; 95\% CI 0.705-2.202], ascites $[P=0.002$; hazard risk (HR), 2.394; 95\% CI 1.394-4.113], hemoglobin < 90g/L $[P=0.001$; hazard risk (HR), 2.674; 95\% CI 1.536-4.655], $\mathrm{LDH} \geq$ $225 \mathrm{U} / \mathrm{L}[P=0.033$; hazard risk (HR), 1.818 ; 95\% CI 1.409-3.150], and 3 times higher level of CEA $[P=0.006$; hazard risk (HR), 2.123; 95\% CI 1.238-3.640] along with CA199 $[P=0.005$; hazard risk (HR), 2.544; 95\% CI 1.332-4.856] than upper limit of normal. The result of multivariate analysis also showed that peritoneal metastasis, hemoglobin level, LDH level, CEA level, and CA199 level were independent prognostic factors for survival (Table 3).

\section{Establishment of the Prognostic Model}

These five independent prognostic factors were included into the model, each representing a risk score of 1 . All the patients were stratified into three groups according to their risk scores: low-risk group (score of 0-1), intermediaterisk group (score of 2-3), and high-risk group (score of 4-5). Of 112 patients, 67 patients were categorized as lowrisk group, 35 patients as intermediate-risk group, and 10 patients as high-risk group. The mOS for low, intermediate, and high-risk groups was 13.9 months ( $95 \%$ CI 10.7-17.1), 8.1 months (95\% CI 5.7-10.4), and 3.9 months (95\% CI 2.6-5.3), respectively, whereas the 1-year survival rate was $56.4 \%, 20.0 \%$, and $0.0 \%$, respectively $(P<$ $0.001)$. The markedly different survival outcomes among the three groups $(P<0.01)$ is shown in Figure 1 .

\section{Discussion}

Chemotherapy is considered one of the effective methods to prolong the OS and improve the life quality of patients with advanced gastric cancer, but the effective rate of chemotherapy is low because of the heterogeneity of the cancer and many other adverse prognostic factors. To this day, there is no single chemotherapy regimen as a standard therapy proven better than others. ${ }^{24}$ Besides, most of the past studies focused on analyzing independent prognostic factors of gastric cancer patients who are candidates for palliative chemotherapy, and few of them put different indicators reflecting poor survival together to analyze the prognosis of those patients. In this study, we analyzed the prognostic factors of 112 advanced gastric cancer patients with fist-line palliative chemotherapy, then designed a convenient prognostic model in order to screen out patients who are more suitable for palliative chemotherapy by the risk stratification.

The PS (ECOG) is the first indicator we need to consider before chemotherapy, because poor PS score has been confirmed an independent risk factor for poor prognosis in patients with advanced gastric cancer before. ${ }^{11,13,22,23}$ However, in our study, the results of univariate analysis show that patients with ECOG score $\geq 2$ had a worse prognosis, but it is not an independent adverse prognostic factor. 
Table I Characteristics of Patients $(n=|| 2)$

\begin{tabular}{|c|c|c|}
\hline Characteristics & Cases & Constituent Ratio (\%) \\
\hline \multicolumn{3}{|l|}{ Age } \\
\hline$\leq 60$ & 72 & 64.3 \\
\hline$>60$ & 40 & 35.7 \\
\hline \multicolumn{3}{|l|}{ Sex } \\
\hline Male & 78 & 69.6 \\
\hline Female & 34 & 30.4 \\
\hline \multicolumn{3}{|l|}{ ECOG status } \\
\hline $0-1$ & 74 & 66.1 \\
\hline 2 & 38 & 33.9 \\
\hline \multicolumn{3}{|l|}{ Pathologic types } \\
\hline Adenocarcinoma & 93 & 83 \\
\hline Signet-ring cell carcinoma & 19 & 17 \\
\hline \multicolumn{3}{|l|}{ Histologic grade } \\
\hline Well differentiated & 2 & 1.8 \\
\hline Moderately differentiated & 23 & 22.3 \\
\hline Poorly differentiated & 69 & 83.9 \\
\hline Unknown & 18 & 16.1 \\
\hline \multicolumn{3}{|l|}{ Number of metastatic organs } \\
\hline$\leq 1$ & 77 & 68.8 \\
\hline$>1$ & 35 & 31.2 \\
\hline \multicolumn{3}{|l|}{ Hepatic metastasis } \\
\hline Yes & 33 & 29.5 \\
\hline No & 79 & 70.5 \\
\hline \multicolumn{3}{|l|}{ Peritoneal metastasis } \\
\hline Yes & 41 & 36.6 \\
\hline No & 71 & 63.4 \\
\hline \multicolumn{3}{|l|}{ Hemoglobin $(g / L)$} \\
\hline$\leq 90$ & 23 & 20.5 \\
\hline$>90$ & 89 & 79.5 \\
\hline \multicolumn{3}{|l|}{ Platelet count $\left(\times 10^{9} / \mathrm{L}\right)$} \\
\hline$\leq 300$ & 79 & 70.5 \\
\hline$>300$ & 33 & 29.5 \\
\hline \multicolumn{3}{|l|}{ LDH (U/L) } \\
\hline$\leq 250$ & 76 & 67.9 \\
\hline$>250$ & 36 & 32.1 \\
\hline \multicolumn{3}{|l|}{ CEA $(\mathrm{ng} / \mathrm{mL})$} \\
\hline$>14.1$ & 69 & 61.6 \\
\hline$\leq 14.1$ & 43 & 38.4 \\
\hline
\end{tabular}

(Continued)
Table I (Continued).

\begin{tabular}{|l|l|l|}
\hline Characteristics & Cases & Constituent Ratio (\%) \\
\hline CA199 (U/mL) & 23 & 18.7 \\
\hline$>81$ & 91 & 81.3 \\
$\leq 81$ & 24 & 21.4 \\
\hline Chemotherapy efficacy & 73 & 65.2 \\
\hline PR & 15 & 13.4 \\
SD
\end{tabular}

Abbreviations: ECOG, Eastern Cooperative Oncology Group; LDH, lactic dehydrogenase; CEA, carcinoembryonic antigen.

Among the advanced gastric cancer patients, peritoneal metastasis is very common and often accompanied by ascites. ${ }^{25}$ Previously, ascites has been reported to be an adverse factor affecting the quality of life and prognosis of patients, ${ }^{11,13}$ and we came to the same conclusion in metastatic gastric cancer patients in the study.

Anemia is also common in patients with advanced gastric cancer, which could be caused by a variety of factors, such as malnutrition, chronic blood loss, postchemoradiotherapy myelosuppression, infiltration of cancer cells in bone marrow, etc. Numerous clinical studies have shown that lower hemoglobin $(\mathrm{Hb})$ is an independent factor for poor prognosis in advanced gastric cancer patients, ${ }^{11,23,26}$ but a few studies come to the conclusion that $\mathrm{Hb}$ does not affect the survival. ${ }^{13}$ In our study we discovered that anemia $(\mathrm{Hb} \leq 90 \mathrm{~g} / \mathrm{L})$ is an adverse factor for predicting survival following first-line palliative chemotherapy in gastric cancer patients with stage IV.

Lactate dehydrogenase (LDH) is a key enzyme that catalyzes the conversion of pyruvate into lactic acid and releases energy during glycolysis. ${ }^{27}$ Because of abnormal supplying vessels, the tumor tissue has remained hypoxic for a long time and its energy supply is mainly dependent on anaerobic glycolysis. ${ }^{28}$ In a number of studies on advanced breast cancer, lung cancer, pancreatic cancer, and lymphoma, high-level serum LDH has been confirmed to signify a poor prognosis. ${ }^{6,15,16,29}$ However, there are relatively few studies on the effect of serum LDH level on the prognosis of advanced gastric cancer. Recently, Namikawa et al reported that the median OS was significantly lower for patients with unresectable advanced gastric cancer who had high $\mathrm{LDH},{ }^{30}$ and we come to the same conclusion in our study. The median OS of patients with 
Table 2 Survival Analysis of Gastric Cancer Patients

\begin{tabular}{|c|c|c|c|}
\hline Characteristics & $\begin{array}{l}\text { mOS } \\
\text { (Months) }\end{array}$ & $\begin{array}{l}\text { I-Year } \\
\text { Survival Rate } \\
\text { (\%) }\end{array}$ & $P$ value \\
\hline \multicolumn{4}{|l|}{ Age: } \\
\hline$\leq 60$ & 9.3 & 35.7 & 0.535 \\
\hline$>60$ & $1 \mathrm{I} .4$ & 38.7 & \\
\hline \multicolumn{4}{|l|}{ Sex: } \\
\hline Male & 10.5 & 42.3 & 0.078 \\
\hline Female & 10.4 & 23.8 & \\
\hline \multicolumn{4}{|l|}{ ECOG status: } \\
\hline $0-1$ & 11.4 & 44.9 & 0.006 \\
\hline 2 & 6.7 & 20.8 & \\
\hline \multicolumn{4}{|l|}{ Pathologic types: } \\
\hline Adenocarcinoma & 11.4 & 42.6 & 0.187 \\
\hline $\begin{array}{l}\text { Signet-ring cell } \\
\text { carcinoma }\end{array}$ & 8.1 & 8.3 & \\
\hline \multicolumn{4}{|l|}{ Histologic grade: } \\
\hline $\begin{array}{l}\text { Well to moderately } \\
\text { differentiated }\end{array}$ & 13.6 & 55.6 & 0.171 \\
\hline Poorly differentiated & 9.3 & 25.0 & \\
\hline \multicolumn{4}{|c|}{ Number of metastatic organs: } \\
\hline$\leq 1$ & 10.4 & 39.6 & 0.600 \\
\hline$>1$ & 10.9 & 32.0 & \\
\hline \multicolumn{4}{|l|}{ Hepatic metastasis } \\
\hline Yes & 9.3 & 34.8 & 0.650 \\
\hline No & 10.6 & 38.0 & \\
\hline \multicolumn{4}{|l|}{ Peritoneal metastasis: } \\
\hline Yes & 6.8 & 17.9 & 0.004 \\
\hline No & 12.2 & 48.9 & \\
\hline \multicolumn{4}{|l|}{ Hemoglobin $(g / L)$} \\
\hline$\leq 90$ & 6.2 & 22.7 & 0.001 \\
\hline$>90$ & II.4 & 43.1 & \\
\hline \multicolumn{4}{|l|}{ Platelet count $\left(\times 10^{9} / \mathrm{L}\right)$} \\
\hline$\leq 300$ & 10.2 & 31.9 & 0.078 \\
\hline$>300$ & 11.5 & 46.2 & \\
\hline \multicolumn{4}{|l|}{$\mathrm{LDH}(\mathrm{U} / \mathrm{L})$} \\
\hline$\leq 250$ & 11.5 & 47.8 & 0.042 \\
\hline$>250$ & 7.4 & 18.5 & \\
\hline
\end{tabular}

(Continued)
Table 2 (Continued).

\begin{tabular}{|c|c|c|c|}
\hline Characteristics & $\begin{array}{l}\text { mOS } \\
\text { (Months) }\end{array}$ & $\begin{array}{l}\text { I-Year } \\
\text { Survival Rate } \\
\text { (\%) }\end{array}$ & $P$ value \\
\hline $\begin{array}{l}>14.1 \\
\leq 14.1\end{array}$ & $\begin{array}{l}6.7 \\
13.6\end{array}$ & $\begin{array}{l}18.8 \\
51.2\end{array}$ & 0.003 \\
\hline \multicolumn{4}{|l|}{ CAI99 (U/mL) } \\
\hline $\begin{array}{l}>81 \\
\leq 81\end{array}$ & $\begin{array}{l}7.4 \\
10.9\end{array}$ & $\begin{array}{l}13.3 \\
43.1\end{array}$ & 0.006 \\
\hline
\end{tabular}

Abbreviations: mOS, median overall survival; ECOG, Eastern Cooperative Oncology Group; LDH, lactic dehydrogenase; CEA, carcinoembryonic antigen.

high-level and low-level serum LDH are 11.5 and 7.4 months, respectively, and the difference is statistically significant $(P=0.042)$. Results from multivariate analysis show that the risk of death in patients with high-level serum LDH is 1.8 times higher than in those with lowlevel serum LDH (95\% CI 1.049-3.150).

The serum CEA and CA199 are the most common tumor markers in gastric cancer, and they show variable levels in different stages. ${ }^{31,32}$ High-level serum CEA and CA199 can predict the recurrence and progression of gastric cancer. Besides, the changes in serum CEA and CA199 levels before and after chemotherapy can predict the efficacy of first-line chemotherapy in advanced gastric cancer. ${ }^{14}$ In our research, patients with high-level serum CEA and CA199 had a shorter survival time, which showed a statistical significance. The results of multivariate analysis indicated that CEA level and CA199 level are independent prognostic factors for metastatic gastric cancer patients treated by first-line chemotherapy.

As far as we know, Lee et al had first established a prognostic model based on 6 clinical factors (no previous gastrectomy, albumin $<3.6 \mathrm{~g} / \mathrm{dL}$, ALP $>85 \mathrm{U} / \mathrm{L}$, performance $\mathrm{ECOG} \geq 2$, presence of bone metastases, and ascites)

Table 3 Results of Multivariate Analysis

\begin{tabular}{|l|l|l|l|}
\hline Characteristics & HR & $\mathbf{9 5 \%} \mathbf{C I}$ & P value \\
\hline ECOG (2) & 1.246 & $0.705-2.202$ & 0.449 \\
Peritoneal metastasis & 2.394 & $1.394-4.113$ & 0.002 \\
Hemoglobin (<90 g/L) & 2.674 & $1.536-4.655$ & 0.001 \\
LDH (>250 U/L) & 1.818 & $1.049-3.150$ & 0.033 \\
CEA (>14.I ng/mL) & 2.123 & $1.238-3.640$ & 0.006 \\
CAI99 (>8I U/mL) & 2.544 & $1.332-4.856$ & 0.005 \\
\hline
\end{tabular}




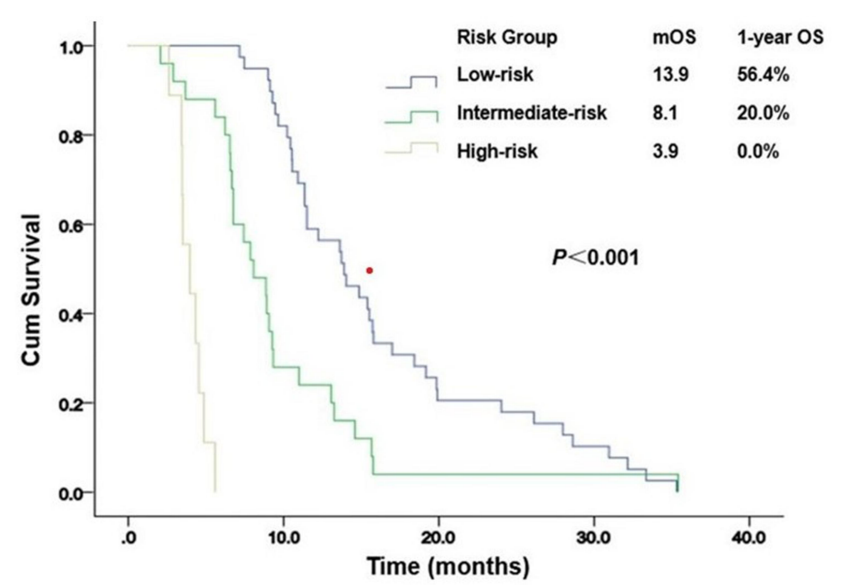

Figure I Survival according to risk groups.

in patients with advanced gastric cancer and used this model to predict survival of these patients undergoing first-line chemotherapy. ${ }^{13}$ The study showed that the median OS for low- and intermediate-risk groups was 12.5 months, but high-risk group showed a median OS of only 2.7 months, which had a significant difference. Then Kanagavel et al established a prognostic model in metastatic gastric cancer patients to predict survival of those following second-line chemotherapy. They found that second-line chemotherapy is not that effective for patients in the high-risk group (with poor PS, lower $\mathrm{Hb}$ level, and worse time to progression) under first-line chemotherapy. ${ }^{23}$ In our research, there is still a significant difference in survival time between the low, medium, and high-risk groups. Patients in the high-risk group have a lower 1-year survival rate among all those patients. The result is similar to those of other researchers. ${ }^{11,22,23}$ As far as our study is concerned, the potential disadvantages are the absence of Lauren's types and a relatively small number of patients. But to the best of our knowledge, the biochemical indicator LDH is included in the prognostic model for the first time as a factor in poor predicted outcome for patients with metastatic gastric cancer.

With the development of medicine, more and more therapeutic methods (such as targeted therapy and immunotherapy, etc.) have been applied to the treatment of patients with advanced gastric cancer. But so far, chemotherapy is still recommended as the primary first-line therapy in NCCN and ESMO guides for advanced gastric cancer patients. Since malignant tumor is a kind of highly heterogeneous disease on a molecular level, individual therapy based on the molecular classification is a progress direction and precision medicine has gradually become a primary treatment of malignant tumors today. Nivolumab and pembrolizumab, two kinds of programmed cell death protein 1 (PD-1) inhibitors, are recommended by the NCCN guide as the third-line therapy in advanced gastric cancer patients that had high levels of PD-L1 expression, which is based on the findings of ATTRACTION-02 and KEYNOTE-059 trials. Besides, based on the KEYNOTE-061 trial, Papbolizumab has been recommended by the NCCN guide for second-line treatment of advanced gastric cancer patients with mismatch repair deficient (dMMR) or microsatellite instability high (MSI-H) molecular subtypes. Recently, the randomized, phase III KEYNOTE-062 trial has achieved its primary endpoint in 2019. The trial shows that first-line pembrolizumab is effective for patients with PD-L1positive, HER2-negative, advanced gastric or gastroesophageal junction (GEJ) cancer, which could provide a new opportunity for people newly diagnosed with advanced gastric or GEJ cancers. Thus, it is expected that in the near future the "right patient" for palliative chemotherapy will be identified not only on the basis of the study of clinical factors, but also on the basis of molecular classification of cases.

\section{Conclusion}

The results of this study show that peritoneal metastasis, hemoglobin level, LDH level, CEA level, and CA199 level are independent prognostic factors for advanced gastric cancer patients receiving first-line palliative chemotherapy, and there are significant differences in the OS between low, intermediate, and high-risk groups $(P<$ 0.001). The simple prognostic model based on these independent prognostic factors could contribute in identifying patient groups which are likely or unlikely to benefit from first-line palliative chemotherapy and making possible the individualized treatment of advanced gastric cancer patients. Chemotherapy or best supportive care- which is better for patients in the high-risk group? This prognosis model may be used as a reference when designing future prospective studies regarding this question.

\section{Ethics}

This study scheme was approved by the ethics committee of Chengdu Seventh People Hospital (batch no: QT2020029-01). As some of the included patients have died before the data collection and all of the data acquired were kept anonymized, our application for exemption from informed 
consent has been approved by the ethics committee of our hospital.

\section{Disclosure}

The authors report no conflicts of interest in this work. Qiyin Zhou and Xi Lan contributed equally to this work and should be considered co-first authors.

\section{References}

1. Bray F, Ferlay J, Soerjomataram I, et al. Global cancer statistics 2018: GLOBOCAN estimates of incidence and mortality worldwide for 36 cancers in 185 countries. CA Cancer J Clin. 2018;68 (6):394-424. doi:10.3322/caac. 21492

2. Wagner AD, Syn NL, Moehler M, et al. Chemotherapy for advanced gastric cancer. Cochrane Database Syst Rev. 2017;8:Cd004064.

3. Bang YJ, Van Cutsem E, Feyereislova A, et al. Trastuzumab in combination with chemotherapy versus chemotherapy alone for treatment of HER2-positive advanced gastric or gastro-oesophageal junction cancer (ToGA): a Phase 3, open-label, randomised controlled trial. Lancet. 2010;376(9742):687-697. doi:10.1016/S0140-6736(10) 61121-X

4. Shitara K, Yatabe Y, Matsuo K, et al. Prognosis of patients with advanced gastric cancer by HER2 status and trastuzumab treatment Gastric Cancer. 2013;16(2):261-267. doi:10.1007/s10120-0120179-9

5. Qiu MZ, Li Q, Wang ZQ, et al. HER2-positive patients receiving trastuzumab treatment have a comparable prognosis with HER2-negative advanced gastric cancer patients: a prospective cohort observation. Int $J$ Cancer. 2014;134(10):2468-2477. doi:10.1002/ijc.28559

6. Sheng WQ, Huang D, Ying JM, et al. HER2 status in gastric cancers: a retrospective analysis from four Chinese representative clinical centers and assessment of its prognostic significance. Ann Oncol. 2013;24(9):2360-2364. doi:10.1093/annonc/mdt232

7. De Carli DM, Rocha MP, Antunes LC, et al. Immunohistochemical expression of HER2 in adenocarcinoma of the stomach. Arq Gastroenterol. 2015;52(2):152-155. doi:10.1590/S000428032015000200015

8. Wagner AD, Grothe W, Haerting J, et al. Chemotherapy in advanced gastric cancer, a systematic review and meta-analysis based on aggregate data. J Clin Oncol. 2006;24(18):2903-2909. doi:10.1200/ JCO.2005.05.0245

9. Yamaguchi K, Yoshida K, Tanahashi T, et al. The long-term survival of stage IV gastric cancer patients with conversion therapy. Gastric Cancer. 2018;21(2):315-323. doi:10.1007/s10120-017-0738-1

10. Lee CM, Choi IK, Kim JH, et al. Is noncurative gastrectomy always a beneficial strategy for stage IV gastric cancer? Ann Surg Treat Res. 2017;92(1):23-27. doi:10.4174/astr.2017.92.1.23

11. Chau I, Norman AR, Cunningham D, et al. Multivariate prognostic factor analysis in locally advanced and metastatic esophago-gastric cancer-pooled analysis from three multicenter, randomized, controlled trials using individual patient data. J Clin Oncol. 2004;22 (12):2395-2403. doi:10.1200/JCO.2004.08.154

12. Yoshida M, Ohtsu A, Boku N, et al. Long-term survival and prognostic factors in patients with metastatic gastric cancers treated with chemotherapy in the Japan Clinical Oncology Group (JCOG) study. Jpn J Clin Oncol. 2004;34(11):654-659. doi:10.1093/jjco/hyh120

13. Lee L, Lim T, Uhm JE, et al. Prognostic model to predict survival following first-line chemotherapy in patients with metastatic gastric adenocarcinoma. Ann Oncol. 2007;18(5):886-891. doi:10.1093/ annonc/mdl501
14. He B, Zhang HQ, Xiong SP, et al. Changing patterns of serum CEA and CA199 for evaluating the response to first-line chemotherapy in patients with advanced gastric adenocarcinoma. Asian Pac J Cancer Prev. 2015;16(8):3111-3116. doi:10.7314/APJCP.2015.16.8.3111

15. Gu J, Zheng L, Wang Y, et al. Prognostic significance of HER2 expression based on trastuzumab for gastric cancer (ToGA) criteria in gastric cancer: an updated meta-analysis. Tumour Biol. 2014;35 (6):5315-5321. doi:10.1007/s13277-014-1693-7

16. Terashima M, Kitada K, Ochiai A, et al. Impact of expression of human epidermal growth factor receptors EGFR and ERBB2 on survival in stage II/III gastric cancer. Clin Cancer Res. 2012;18 (21):5992-6000. doi:10.1158/1078-0432.CCR-12-1318

17. Shen GS, Zhao JD, Zhao JH, et al. Association of HER2 status with prognosis in gastric cancer patients undergoing R0 resection: a large-scale multicenter study in China. World $J$ Gastroenterol. 2016;22(23):5406-5414. doi:10.3748/wjg.v22.i23.5406

18. Qiu M, Zhou Y, Zhang X, et al. Lauren classification combined with HER2 status is a better prognostic factor in Chinese gastric cancer patients. BMC Cancer. 2014;14(1):823. doi:10.1186/1471-2407-14-823

19. Kurokawa Y, Matsuura N, Kimura Y, et al. Multicenter large-scale study of prognostic impact of HER2 expression in patients with resectable gastric cancer. Gastric Cancer. 2015;18(4):691-697. doi:10.1007/s10120-014-0430-7

20. Janjigian YY, Werner D, Pauligk C, et al. Prognosis of metastatic gastric and gastroesophageal junction cancer by HER2 status: a European and USA International collaborative analysis. Ann Oncol. 2012;23(10):2656-2662.

21. Hecht JR, Bang YJ, Qin SK, et al. Lapatinib in combination with capecitabine plus oxaliplatin in human epidermal growth factor receptor 2-positive advanced or metastatic gastric, esophageal, or gastroesophageal adenocarcinoma: trio-013/logic-a randomized phase III trial. J Clin Oncol. 2016;34(5):443-451. doi:10.1200/ JCO.2015.62.6598

22. Catalano V, Graziano F, Santini D, et al. Second-line chemotherapy for patients with advanced gastric cancer: who may benefit? $\mathrm{Br}$ $J$ Cancer. 2008;99(9):1402-1407. doi:10.1038/sj.bjc.6604732

23. Kanagavel D, Pokataev IA, Fedyanin MY, et al. A prognostic model in patients treated for metastatic gastric cancer with second-line chemotherapy. Ann Oncol. 2010;21(9):1779-1785. doi:10.1093/ annonc/mdq032

24. Ge XY, Ge F, Wang Z, et al. Analysis of risk factors of stage IV gastric cancer from the SEER database. Ann $R$ Coll Surg Engl. 2020;102(5):355-362. doi:10.1308/rcsann.2020.0031

25. Ishigami $\mathrm{H}$, Omori $\mathrm{T}$, Fukushima R, et al. Long-term normothermic intraperitoneal and systemic chemotherapy for gastric cancer with peritoneal metastasis: results from clinical trials over a decade in Japan. Eur J Surg Oncol. 2019;45(2):e6-e7. doi:10.1016/j.ejso.2018.10.119

26. Inal A, Kaplan MA, Kuçukoner M, et al. Prognostic factors in first-line chemotherapy treated metastatic gastric cancer patients: a retrospective study. Asian Pac J Cancer Prev. 2012;13 (8):3869-3872. doi:10.7314/APJCP.2012.13.8.3869

27. Wu XT, Liu JQ, Lu XT, et al. The enhanced effect of lupeol on the destruction of gastric cancer cells by NK cells. Int Immunopharmacol. 2013;16(2):332-340. doi:10.1016/j.intimp.2013.04.017

28. Chen F, Chen J, Yang L, et al. Extracellular vesicle-packaged HIF$1 \alpha$-stabilizing IncRNA from tumour-associated macrophages regulates aerobic glycolysis of breast cancer cells. Nat Cell Biol. 2019;21(4):498-510. doi:10.1038/s41556-019-0299-0

29. Chung KM, Chang ST, Huang WT, et al. Bcl-6 expression and lactate dehydrogenase level predict prognosis of primary gastric diffuse large B-cell lymphoma. J Formos Med Assoc. 2013;112 (7):382-389. doi:10.1016/j.jfma.2012.07.031

30. Namikawa T, Ishida N, Tsuda S, et al. Prognostic significance of serum alkaline phosphatase and lactate dehydrogenase levels in patients with unresectable advanced gastric cancer. Gastric Cancer. 2019;22(4):684-691. doi:10.1007/s10120-018-0897-8 
31. Shimada $\mathrm{H}$, Noie $\mathrm{T}$, Ohashi $\mathrm{M}$, et al. Clinical significance of serum tumor markers for gastric cancer, a systematic review of literature by the task force of the Japanese Gastric Cancer Association. Gastric Cancer. 2014;17(1):26-33.
32. Kim DH, Oh SJ, Oh CA, et al. The relationships between perioperative CEA, CA19-9, and CA72-4 and recurrence in gastric cancer patients after curative radical gastrectomy. J Surg Oncol. 2011;104 (6):585-591. doi:10.1002/jso.21919

\section{Publish your work in this journal}

Cancer Management and Research is an international, peer-reviewed open access journal focusing on cancer research and the optimal use of preventative and integrated treatment interventions to achieve improved outcomes, enhanced survival and quality of life for the cancer patient.
The manuscript management system is completely online and includes a very quick and fair peer-review system, which is all easy to use. Visit http://www.dovepress.com/testimonials.php to read real quotes from published authors. 